\title{
Screening for Vision Impairments in Individuals with Dementia Living in Long-Term Care: A Scoping Review
}

\author{
Jennifer L. Campos ${ }^{\mathrm{a}, \mathrm{b}, *}$, Fiona Höbler ${ }^{\mathrm{a}, \mathrm{c}}$, Etty Bitton ${ }^{\mathrm{d}}$, Tammy Labreche ${ }^{\mathrm{e}}$, \\ Katherine S. McGilton ${ }^{\mathrm{a}, \mathrm{f}}$ and Walter Wittich ${ }^{\mathrm{d}, \mathrm{g}}$ \\ ${ }^{a}$ Toronto Rehabilitation Institute, University Health Network, Toronto, ON, Canada \\ ${ }^{\mathrm{b}}$ Department of Psychology, University of Toronto, Toronto, ON, Canada \\ ${ }^{\mathrm{c}}$ Rehabilitation Sciences Institute, University of Toronto, Toronto, ON, Canada \\ dÉcole d'optométrie, Université de Montréal, Montréal, Québec, Canada \\ ${ }^{\mathrm{e}}$ School of Optometry and Vision Science, University of Waterloo, Waterloo, ON, Canada \\ ${ }^{\mathrm{f}}$ Lawrence S. Bloomberg Faculty of Nursing, University of Toronto, Toronto, ON, Canada \\ ${ }^{\mathrm{g}}$ Center for Interdisciplinary Rehabilitation Research of Greater Montreal, Montréal, Québec, Canada
}

Handling Associate Editor: Manuel Montero-Odasso

Accepted 21 January 2019

\begin{abstract}
Vision impairments are prevalent, but underdiagnosed in individuals with dementia living in long-term care (LTC). Effective screening tools could identify remediable vision problems. This scoping review was conducted to identify vision screening tests used with individuals with dementia and assesses their suitability for administration by nurses in LTC. A literature search using the Arksey and O'Malley (2005) method included research articles, conference proceedings, and dissertations. Data were included from participants over 65 years of age with a diagnosis of probable dementia. A panel of vision experts evaluated the suitability of the candidate vision tests. The search yielded 179 publications that met the inclusion criteria. Of 134 vision tests that were identified, 19 were deemed suitable for screening by nurses in LTC. Tests screened for acuity (12), visual field (1), anatomy (2), color vision (2), and general visual abilities (2). Tests were excluded because of complexity of interpretation (90), need for specialized training (83), use in research only (57), need for specialized equipment (54), not assessing visual function (44), long test duration (21), uncommonness (13), and needing an act reserved for specialists (7). Psychometric properties were not often reported for tests. Few of the tests identified had been validated for use with individuals with dementia. Based on our review, few tests were deemed suitable for use by nurses to assess this population in LTC. Identifying appropriate tools to screen vision in individuals with dementia is a necessary first step to interventions that could potentially improve functioning and quality of life.
\end{abstract}

Keywords: Aging, cognition, decline, low vision, nursing, sensory

\section{INTRODUCTION}

Visual impairments in individuals with dementia

${ }^{*}$ Correspondence to: Jennifer Campos, $\mathrm{PhD}, 550$ University Ave., Toronto, ON, M5G 2A2, Canada. E-mail: Jennifer.Cam pos@uhn.ca.

Visual impairments can have a profound effect on critical everyday functions such as mobility, communication, and participation. Visual impairments 
are associated with greater incidence of depression, anxiety, loneliness, reduced quality of life, reduced participation, and increased risk of falls [1-5]. Declines in vision become more prevalent with advancing age and are particularly common in long-term care (LTC), with an estimated prevalence of $30-57 \%$ [6-8]. There is also an increased prevalence of cognitive impairments in LTC residents, with $60-80 \%$ of residents diagnosed with dementia [9]. In older individuals with cognitive impairments, it is estimated that $30-51 \%$ have both cognitive and visual impairments [9-11]. Because there are challenges in assessing vision in individuals with dementia, these numbers may underestimate the prevalence of vision impairments in this population.

The combination of sensory and cognitive impairment can lead to poorer health outcomes than would result from either impairment alone [11, 12], including increased mobility limitations, problems performing activities of daily living [12], and poorer communication [11]. While the nature of the associations between visual and cognitive abilities is not entirely clear (and is likely bidirectional), increased risk of cognitive decline has been linked to visual impairments [13-21]. In this paper, we focus specifically on screening vision in individuals who have been diagnosed with dementia to identify those who might benefit from interventions that could potentially improve functioning and quality of life in the face of coexisting sensory and cognitive impairments.

It is possible that untreated visual impairments in individuals with dementia could 1) evoke or exacerbate symptoms such as depression, anxiety, loneliness, or aggression [22]; 2) contribute to impaired performance on tasks requiring cognitive executive functioning or memory due to increased cognitive load [23]; and/or 3) result in accelerated rates of cognitive decline [13]. Individuals with dementia may also respond to vision loss differently than those without (e.g., be more prone to visual hallucinations [19, 24] or time-of-day effects [25]). There is encouraging evidence to suggest that treating visual impairments can improve the quality of life of individuals with mild or moderate cognitive impairments [19, 26-28], but the effects of interventions on individuals with dementia have not been well investigated.

Overall, very little is known about the optimal way to screen visual abilities in individuals with dementia, particularly within an LTC setting [29]. In an effort to tackle this problem, our team has employed a mixed-methods approach to identify and evalu- ate existing screening tools and approaches. In this paper, we present the first phase of this project, which consisted of a scoping review of the scientific literature describing: 1) visual abilities that have been screened in individuals with dementia (e.g., acuity, visual field);2) approaches and tools used to test these abilities; 3) psychometric properties of these tools; and 4) suitability of these tools for use with individuals with dementia. An overview of the necessary considerations for performing vision screening with this population and in the LTC setting is described below.

\section{Vision screening in individuals with dementia living in LTC}

Despite the significant potential importance of identifying visual impairments in individuals with dementia living in LTC, it is not without its challenges. Routine assessments of every LTC resident by formally trained vision specialists are often not efficient or feasible [37]. The ability to travel off-site for professional vision assessments can be hampered by the reduced mobility of the residents and the time and resources required of their care providers. An alternative would be screening of residents on-site, within their care facilities, by members of their care team (i.e., nurses), resulting in referrals to vision care specialists for full assessments as required [30]. To optimize the screening of visual impairments in individuals with dementia living in LTC, there are several categories of unique factors to consider, including those attributable to the individual (i.e., dementia diagnosis), the test administrator (i.e., nurses), and the context (i.e., LTC). We briefly address these factors below.

\section{Vision screening in individuals with dementia}

Ideally, in order to ensure that vision screening tools are accurately and reliably measuring visual impairments in individuals with dementia, the test outcomes should not be confounded by co-existent cognitive impairment. The task or instructions should not unnecessarily tax working memory, executive functioning, or attention span [31]. In other words, it is important to ensure that a positive result on a screening test for vision impairment is truly due to sensory loss and not due to an inability to comprehend or perform the test procedures. Recommended strategies for minimizing confounds due to cognitive impairment include using concise directions, repeating instructions, speaking clearly, providing 
encouragement, and limiting the time required to complete the test [24, 30, 31].

Similarly, a high proportion of individuals over 75 years of age have hearing loss (80\% [32]), with hearing loss being more prevalent in individuals with dementia than in those with normal cognition [33-35]. Therefore, to ensure that information presented auditorily is heard as well as possible, it is important to administer tests in a quiet room, using assistive hearing devices if necessary, and whenever possible to supplement auditory information with visual information and to minimize competing cognitive demands (e.g., by talking face-to-face with adequate lighting and minimal distractions). While standardized questionnaires or intake histories (e.g., Activities of Daily Living Scale, informal inquiries about use of glasses, or reported history of ocular surgeries) may be useful, the reliability of self-reports may be questionable, particularly for those at more advanced stages of dementia [31]. Therefore, soliciting comments from family, friends, or caregivers about their observations of vision-related functioning is advisable [38]. It is also important to consider the functional significance of potentially improving different visual abilities and the use of screening to help prioritize abilities that could best improve quality of life. For instance, if the goal for the older adult is to improve mobility, social interactions, or reading, then screening for acuity or visual field may be more important than screening for color vision. Developing vision-related quality of life and activity of daily living inventories has been proposed to identify functional benefits associated with the remediation of vision problems [24]. Overall, the largest gap in knowledge is that very few existing screening tools have been intentionally customized, optimized, and validated for use with individuals with dementia living in LTC $[36,37]$.

\section{Administration of vision screening by nurses}

A significant challenge to nurses' abilities to screen for vision impairments in their clients is limited time and resources [38]. A screening tool should be quick and intuitive to administer. It must be readily available, with little need for calibration or complex equipment, and little training should be required to interpret the results. Nurses must feel qualified to conduct the screening measures using standard procedures and be confident in their ability to interpret the results. There must also be a clear procedure for nurses to follow when making referrals to vision specialists.
Given their daily interactions and intimate familiarity, nurses are often uniquely prepared to interpret their clients' responses during sensory testing and may have a particularly keen awareness of even subtle behavior or changes in behavior. They may also be best able to identify the ideal time to administer a test based on their client's daily patterns/routines and present state of mind. These advantages may not be realized as fully by healthcare providers who have not already established a relationship with the client, such as vision specialists from clinics external to the LTC setting.

\section{Screening vision within an LTC setting}

LTC facilities are typically not equipped with dedicated sensory assessment tools or technologies [39]. Equipment/materials for screening must be inexpensive, compact, light, portable and durable, and must be easy to maintain, disinfect, and repair. Appropriate space must be available for testing, including a comfortable space with limited background noise and appropriate lighting levels. There must also be commitment by nursing managers and administrative staff to prioritize vision screening, including arranging for the provision of training, communicating the importance of vision screening to staff, and supporting the protocols for referral [30].

\section{Research questions and project objectives}

This scoping review was conducted to provide a comprehensive review of the measures used in the scientific literature to screen for visual impairments in older adults with dementia. The search helped inform and was subsequently supplemented by two environmental scans, one with vision specialists (e.g., ophthalmologists and optometrists [31]) and a second with nurses working in LTC [30]. The results of the scoping review described in this paper and the environmental scans were used to inform a Delphi panel of experts in their selection of vision measures to be tested for feasibility and reliability of use by nurses working with individuals with dementia in LTC [31]. Overall, the goals of this comprehensive series of studies were to 1) summarize the approaches used for vision screening for those with dementia living in LTC; 2) identify gaps in scientific knowledge to motivate future research; 3 ) identify gaps in current clinical practice to motivate enhanced vision screening; and 4) use this composite knowledge to develop novel sensory screening tools that are ide- 
Table 1

Procedure and exclusion codes for citation review

\begin{tabular}{|c|c|c|}
\hline Code & Criteria & Rationale for excluding papers \\
\hline 1 & Participants & $\begin{array}{l}\text { Participants in study are neither diagnosed with dementia nor classed as having cognitive impairment by } \\
\text { MMSE score <24; participants are not human (e.g., mice, primates) } \\
\text { note: reports about Parkinson's disease were excluded, unless specified as Parkinson's disease dementia }\end{array}$ \\
\hline 2 & No vision & Study does not use vision impairment assessment tools/instruments, e.g.: visual attention task \\
\hline 3 & Age & Participants $<65$ years of age \\
\hline 4 & Report type & Report is an editorial, review, expert opinion, book \\
\hline 5 & No abstract & Report cannot be judged due to missing abstract \\
\hline 6 & Duplication & More than one citation of same article \\
\hline 7 & Publication year & $<1995$ \\
\hline [blank] & Include & Vision assessment in neurodegenerative participants $\geq 65$ years of age \\
\hline
\end{tabular}

ally suited for administration by nurses to residents with dementia living in LTC.

\section{METHODS}

\section{Scoping review}

This scoping review followed the protocol outlined by McGilton et al. [29]. The methodological framework by Arksey and O'Malley [40] was used to guide a literature search of all relevant research, regardless of study design, by iterative and reflexive means. Five stages of review were completed to: 1) identify the research question, 2) identify relevant studies, 3) select the studies for inclusion, 4) chart the data, and 5) summarize and report the results.

\section{Identifying the research question}

A broad search strategy with clearly defined concepts was employed [40], whereby search terms were continuously refined by group consensus within a multidisciplinary team of reviewers [29]. The team engaged in group consultation to inform the search criteria and clinical applicability of data for extraction, and to allow for post-hoc development of inclusion and exclusion criteria and data synthesis in terms of the value yielded by qualitative or quantitative analyses of results.

Screening measures were defined as either objective or subjective tests and instruments that were appropriate for use in the preliminary evaluation of visual abilities or the detection (i.e., screening) of vision loss, including paper-based tests and test technologies that could be software in the form of apps or portable ophthalmic equipment. Assessments used for diagnostic purposes were also initially captured in the search; however, these were excluded at a later stage of the review process given that this scoping review and test evaluation was focused on screening tools rather than diagnostic tools.

\section{Identifying relevant studies}

Electronic databases of Embase, Medline, PsycINFO, CENTRAL, and CINAHL were searched by an Information Specialist at the Toronto Rehabilitation Institute-University Health Network (JB), using both subject headings (Mesh, Emtree) and keywords to search for the concepts of vision tests, long-term care settings, and neurodegenerative diseases in studies published between 1995 and 2017 [29]. Based on the initial search results from several trialed database searches, a recursive process of revising and extending key search terms by definition was used and adapted to those appropriate to each database (Supplementary Material).

Peer-reviewed and non-peer-reviewed publications included quantitative and qualitative research articles, assessment and treatment studies, conference proceedings, and academic dissertations. All publications reported original data from participant groups with a mean or median age of 65 years or older and with neurodegenerative disease. Further post-hoc refinements were applied to the search criterion of neurodegenerative disease to only include articles describing individuals with dementia and/or Alzheimer's disease. No language restrictions were applied.

Each title and abstract was independently screened by two reviewers with expertise in vision, nursing, psychology, and/or speech-language pathology. Reviewers coded articles for exclusion with the first code that applied from (Table 1). Discrepancies were arbitrated by a third reviewer or through consensus discussion between the original two reviewers.

Through group consultation, an Excel spreadsheet was trialed and standardized for data extraction pur- 
poses. The following information, if reported in the paper, was extracted from the 179 included articles: author information, year of publication, country in which the research was performed, testing environment (e.g., clinic, research laboratory, LTC), study design, sampling method, participant demographics (age, gender, dementia type, comorbidities), name of the screening/assessment tool(s), abilities tested, time required to complete testing, number of incomplete tests, adaptations made for clinical populations, measurement outcomes, interpretation of results, psychometric properties (validity, reliability, sensitivity, specificity), and integrity of administration (who administered and interpreted the test). Collated data were proofread for accuracy and consistency.

\section{Expert panel evaluations of suitability}

Members of the review team with clinical experience in the use of vision screening measures (two optometrists and one certified low vision therapist) evaluated suitability for each of the identified vision tests. Suitability was defined by the test's efficiency and effectiveness of purpose in the specified screening context (i.e., screening vision in individuals with dementia in LTC), as well as the acceptability of its content and interpretation by nurses in LTC [29, 41]. The evaluation of suitability also included consideration of each tool's determinants of sensibility. Determining sensibility is an important pre-requisite to examining the reliability and validity of an instrument, and is critical to its acceptance by nurses and actual utility in testing the intended population [29, 41]. Suitability was determined using the following exclusion criteria: reserved acts limited to the scope of practice of eye care professionals only (e.g., refraction testing), specialized equipment required, specialized training required, interpretation too complex, duration too lengthy, not commonly used in practice, not a test of visual function, or only used for research purposes. After independently reviewing every unique vision test, all three specialists met once via teleconference to resolve any disagreements and come to a consensus regarding suitability for each candidate test.

\section{RESULTS}

\section{Scoping review}

A total of 3,716 research citations were found across three stages of database searches conducted on the 6 November 2015, 23 June 2016, and 10 February 2017 (See Fig. 1 for the PRISMA flow diagram). After a review based on title and abstract, 280 research articles were selected for a full paper review. Each article was independently reviewed in full by two reviewers, who further excluded 101 citations. There were 179 citations agreed upon by consensus to be included in the review and undergo data extraction (Supplementary Table 1).

\section{Expert panel evaluations of suitability}

Table 2 provides a summary of the 19 screening tools that were judged to be suitable based on all of the criteria described above. These tools included two tests of anatomy and physiology, six tests of near visual acuity, six tests of distance visual acuity, one test of visual field, two tests of color vision, and two self-report questionnaires related to activities of daily living and visual function. Supplementary Table 2 provides a summary of the 115 screening tools that were deemed unsuitable, including 12 tests of anatomy and physiology, five of near visual acuity, five of distance visual acuity, 10 of eye movements, three of refraction, seven of visual field, seven of color vision, 10 of contrast sensitivity, one of critical flicker fusion, 11 questionnaires, two visual perceptual tests, and 42 other tests considered to be uncommon in standard practice by vision specialists. Of all the tests identified (suitable and unsuitable), the frequency with which specific psychometric properties were described across all included studies were: reliability (18), validity (22), sensitivity and/or specificity (16), and sensibility (73). The specific frequency for each type of psychometric property for the 19 screening tools judged suitable is provided in (Table 3 ).

\section{DISCUSSION}

\section{Summary of the literature}

In summary, the number of publications that met inclusion criteria was 179; the total number of times a vision test was mentioned or described within these publications was 383; of those mentions, the number of unique individual vision tests identified was 134 , of which 19 tests were deemed to be suitable based on expert review (the remaining 115 tests deemed unsuitable). Overall, the results of this scoping review suggest that few tools used in the reviewed literature (only about 14\%) were deemed suitable for 


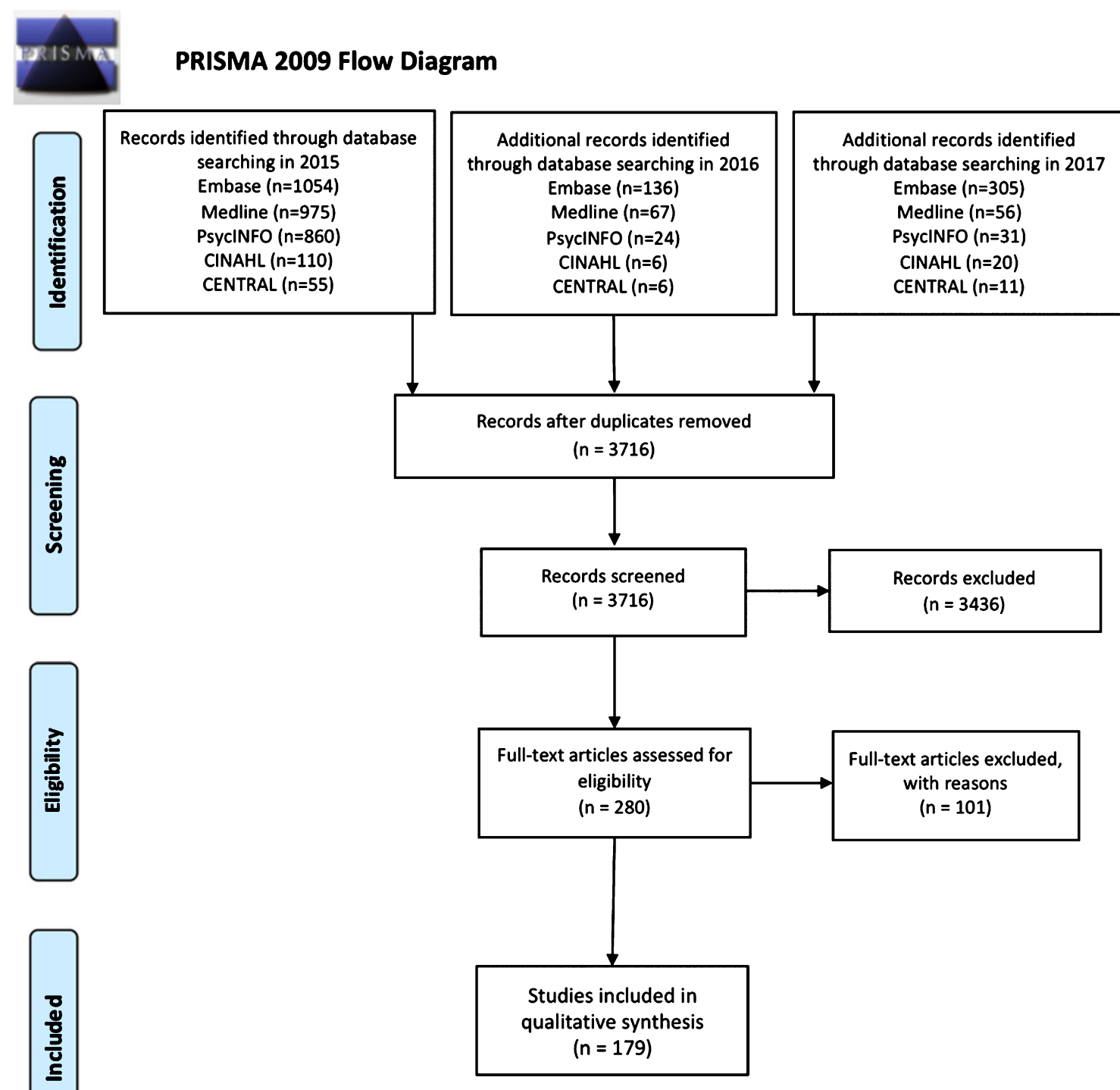

From: Moher D, Liberati A, Tetzlaff J, Altman DG. The PRISMA Group (2009). Preferred Reporting Hems for Systematic Reviews and Meta-Analyses: The PRISMA Statement. PLoS Med 6(7): e1000097. doi:10.1371/journal.pmed1000097

For more information, visit www.prisma-statement.org.

Fig. 1. PRISMA flow diagram of included and excluded studies.

screening vision in individuals with dementia living in LTC. Of the measures that met the inclusion criteria, most screened for visual acuity (near [6] and distance [6]), one screened for visual field, and two others each screened for anatomy, color vision, and for visual abilities via questionnaires. Reasons for the exclusion of tests included: overly complex to interpret results (90), need for specialized training (83), research use only (57), need for specialized equipment (54), visual function not assessed (44), duration overly long (21), not commonly used (13), requirement of an act reserved for vision specialists (7). The majority of studies did not report on the psychometric properties of the test or comment on any barriers to use or adjustments made for testing individuals with dementia. 
Table 2

Vision tests deemed suitable for screening older adults with dementia in LTC

\begin{tabular}{|c|c|c|c|}
\hline Category of test & Name of test & Ability tested & $\begin{array}{c}\text { Citation } \\
\text { Frequency }\end{array}$ \\
\hline \multirow[t]{2}{*}{ Anatomy/Physiology } & $\begin{array}{l}\text { Neuro-ophthalmic } \\
\text { Assessment }\end{array}$ & $\begin{array}{l}\text { Ocular movements, pupils, ductions R (right) \& L (left) and } \\
\text { versions; e.g., conversion; assesses neurological function, cranial } \\
\text { nerve III, IV, VI) }\end{array}$ & 15 \\
\hline & Pupil Reflex & $\begin{array}{l}\text { Observe if pupils constrict equally, neurological evaluation for } \\
\text { cranial nerve II (need pen light or transilluminator) }\end{array}$ & 9 \\
\hline \multirow[t]{6}{*}{ Visual Acuity - near } & Counting Fingers & $\begin{array}{l}\text { Cranial nerve II and macular function (must be administered } \\
\text { monocularly) }\end{array}$ & 1 \\
\hline & Hand Motion & $\begin{array}{l}\text { Cranial nerve II and macular function (must be administered } \\
\text { monocularly) }\end{array}$ & 1 \\
\hline & Acuity card (letters) & $\begin{array}{l}\text { Near vision, preferable with habitual refraction (with their glasses } \\
\text { on), under good lighting and a glare-free condition }\end{array}$ & 4 \\
\hline & $\begin{array}{l}\text { Lighthouse Near Visual } \\
\text { Acuity Test (Modified } \\
\text { ETDRS) [48] }\end{array}$ & $\begin{array}{l}\text { Near vision @ } 40 \mathrm{~cm} \text {, preferable with habitual refraction (with their } \\
\text { glasses on), under good lighting and a glare-free condition }\end{array}$ & 8 \\
\hline & $\begin{array}{l}\text { Rosenbaum Pocket } \\
\text { Vision Screener }\end{array}$ & $\begin{array}{l}\text { Portable near vision acuity card, preferable with habitual refraction } \\
\text { (with their glasses on), under good lighting and a glare-free } \\
\text { condition }\end{array}$ & 2 \\
\hline & Patty pics & Pictures of face, uses preferential looking technique & 2 \\
\hline \multirow[t]{6}{*}{ Visual Acuity - distance } & Acuity chart (letters) & $\begin{array}{l}\text { Distance vision, preferable with habitual refraction (with their } \\
\text { glasses on), under good lighting and a glare-free condition, } \\
\text { monocular ideal }\end{array}$ & 2 \\
\hline & Snellen chart (letters) [49] & $\begin{array}{l}\text { Distance vision, preferable with habitual refraction (with their } \\
\text { glasses on), under good lighting and a glare-free condition, } \\
\text { monocular ideal }\end{array}$ & 30 \\
\hline & $\begin{array}{l}\text { HOTV chart }(\mathrm{h}, \mathrm{o}, \mathrm{t}, \mathrm{v} \\
\text { letters) }\end{array}$ & $\begin{array}{l}\text { Distance vision, preferable with habitual refraction (with their } \\
\text { glasses on), under good lighting and a glare-free condition, } \\
\text { monocular ideal }\end{array}$ & 1 \\
\hline & Landolt C chart (C's) [50] & $\begin{array}{l}\text { Distance vision, preferable with habitual refraction (with their } \\
\text { glasses on), under good lighting and a glare-free condition, } \\
\text { monocular ideal }\end{array}$ & 6 \\
\hline & Tumbling E chart (E's) & $\begin{array}{l}\text { Distance vision, preferable with habitual refraction (with their } \\
\text { glasses on), under good lighting and a glare-free condition, } \\
\text { monocular ideal }\end{array}$ & 2 \\
\hline & Feinbloom & $\begin{array}{l}\text { Distance vision, preferable with habitual refraction (with their } \\
\text { glasses on), under good lighting and a glare-free condition, } \\
\text { monocular ideal }\end{array}$ & 2 \\
\hline $\begin{array}{l}\text { Visual Field } \\
\end{array}$ & Confrontation fields & $\begin{array}{l}\text { Visual field face to face using hands and objects, required training } \\
\text { for interpretation }\end{array}$ & 4 \\
\hline \multirow[t]{2}{*}{ Color vision - near } & Ishihara plates [51] & $\begin{array}{l}\text { Color test, presence of congenital (binocular testing) or acquired } \\
\text { (monocular testing) color impairments, requires test plates, } \\
\text { suitable lighting }\end{array}$ & 4 \\
\hline & $\begin{array}{l}\text { City University Colour } \\
\text { Vision test [52] }\end{array}$ & Color matching test to detect congenital color deficiencies & 5 \\
\hline \multirow[t]{2}{*}{ Questionnaire } & $\begin{array}{l}\text { Activities of Daily Living } \\
\text { Assessment Scale } \\
\text { (ADL scale) }\end{array}$ & Subjective perception of visual problems & 1 \\
\hline & $\begin{array}{l}\text { National Eye Institute } \\
\text { Visual Function } \\
\text { Questionnaire [53] }\end{array}$ & Subjective perception of visual problems & 1 \\
\hline
\end{tabular}

To our knowledge, the only study reporting a customized screening measure for individuals with dementia living in LTC was one used to screen for visual acuity [37]. In that study in which customized screening protocols were implemented, $85.3 \%$ of residents were identified as needing a full vision assessment by a clinical vision specialist. This high number highlights both the importance of screening individuals with dementia in LTC, but also the practical issues associated with a very high rate of those who fail screening. A recent review indicated that routine vision care is lacking within the LTC setting [42]. Rather than being triggered by LTC staff, the provision of eye care is typically initiated through 
Table 3

Summary of psychometric properties reported for the suitable tests. Checkmarks provide a frequency count for each time the psychometric property represented in each column was reported for each suitable test across studies

\begin{tabular}{|c|c|c|c|c|}
\hline & Reliability & Validity & $\begin{array}{c}\text { Sensitivity \& } \\
\text { Specificity }\end{array}$ & Sensibility \\
\hline Total Articles that provide & 18 & 22 & 16 & 73 \\
\hline Neuro-ophthalmic assessment & & & $\checkmark$ & $\checkmark \checkmark$ \\
\hline \multicolumn{5}{|l|}{ Pupil reflex } \\
\hline Counting fingers & & & & $\checkmark$ \\
\hline Hand motion & & & & $\checkmark$ \\
\hline \multicolumn{5}{|l|}{ Acuity card (letters) } \\
\hline Lighthouse near visual acuity test & $\checkmark$ & $\checkmark$ & & \\
\hline Rosenbaum pocket vision screener & & & & $\checkmark$ \\
\hline Patty pics & & $\checkmark$ & & $\checkmark$ \\
\hline Acuity chart (letters) & $\checkmark$ & $\checkmark \checkmark \checkmark$ & $\checkmark$ & $\checkmark \checkmark \checkmark$ \\
\hline Snellen chart (letters) & & $\checkmark \checkmark$ & & $\checkmark \checkmark \checkmark \checkmark \checkmark \checkmark \checkmark \checkmark$ \\
\hline HOVT chart (h, o, t, v letters) & & $\checkmark$ & & \\
\hline Landolt C chart (c's) & $\checkmark$ & $\checkmark$ & $\checkmark \checkmark$ & \\
\hline \multicolumn{5}{|l|}{ Tumbling e chart (e's) } \\
\hline \multicolumn{5}{|l|}{ Feinbloom } \\
\hline Confrontation fields & & & & $\checkmark \checkmark$ \\
\hline Ishihara plates & & & & $\checkmark \checkmark$ \\
\hline City university colour vision test & $\checkmark$ & $\checkmark \checkmark$ & & \\
\hline Activities of Daily Living assessment scale & & & & \\
\hline National Eye Institute Visual Function Questionnaire & $\checkmark \checkmark$ & $\checkmark$ & & \\
\hline
\end{tabular}

requests by the individuals themselves or their families [42]. Individuals who are less able to self-identify impairments or to self-advocate are at greater risk of being overlooked in the absence of routine screening.

\section{Outcomes of vision screening}

Evidence that screening is effective at identifying previously unrecognized impairments would strongly motivate the implementation of routine use of efficient vision screening. While evidence regarding the effectiveness of vision screening is limited, there is some indication that vision screening would identify a large proportion of individuals who might potentially benefit from vision interventions. For instance, in the PrOVIDe study (Prevalence Of Visual Impairment in people with Dementia), Bowen et al. [10] reported that of individuals identified as having a visual impairment, $47 \%$ had vision problems that could be corrected through prescription lenses and almost $50 \%$ of those with impairments that were not corrected by lenses had impairments that could be corrected through cataract surgery. Notably, uncorrectable visual impairments were higher in individuals with dementia living in LTC and there were more difficulties assessing vision within this group ( $34.2 \%$ could not be assessed) compared to individuals with dementia living at home $(2.6 \%$ could not be assessed). It is possible that with improved vision screening measures, the number of individuals who could be assessed in LTC may increase. Jee et al. [43] demonstrated that of older individuals transitioning to LTC who were screened for impairments in visual acuity, 18-20\% could improve their vision with modifications to their prescription lenses. Chriqui et al. [9] indicated that, of individuals with dementia living in LTC with a visual impairment, $40 \%$ could benefit from adjusted prescriptions. Thus, this evidence suggests that there are a significant number of individuals with dementia who are living with correctable visual impairments who could benefit from enhanced screening measures and protocols.

Of course, the key benefit of identifying visual impairments is to then remediate correctable problems, thereby improving everyday functioning and quality of life. While there is limited knowledge about these outcomes, Owsely et al. [6, 28] investigated whether updating eyeglass prescriptions and removing cataracts were associated with measurable benefits to those with dementia. They demonstrated that such interventions reduced depression and improved quality of life, reading abilities, and social interactions. Therefore, while there is still much to be learned about how best to screen for vision impairment in this population, the existing evidence is encouraging and suggests that vision screening could lead to remediation that could be potentially beneficial. 


\section{Limitations and identified priorities for future} directions

A limitation with a scoping review of the scientific literature is that it does not necessarily reflect the measurements that are used in actual practice or what adaptive strategies are applied when tests are used in practice. This is where environmental scans provide additional qualitative data surrounding the use of tools in practice [30, 31]. Further, there were many differences across studies with respect to the severity and type of dementia, the age of participants, the specialization of the person administering the test, and the setting within which it was administered. The majority of studies included individuals with Alzheimer's disease of mild-moderate severity, and tests were typically conducted in a clinical environment. Severity of dementia is likely to be an important factor associated with the feasibility and utility of using and adapting vision screening measures in practice and the potential benefits of remediation based on screening conducted by LTC staff. It will be especially important to learn about the use of vision screening measures in individuals at more severe stages of dementia. Even if benefit from personal solutions diminishes as the severity of dementia increases, environmental modifications or adaptive strategies used by care providers and family may nevertheless still be beneficial.

As individuals age, there is not only increased risk of vision loss and cognitive decline, but there are often additional declines in other sensory functions, including hearing [11, 44-47]. The studies reported within this scoping review primarily focused on visual impairment, but it is likely that a large number of the participants in the reviewed studies also experienced age-related hearing loss. While a few studies reported on dual-sensory loss (around 10\% of the initial search and 16 of the accepted studies), most studies did not report on hearing abilities. Therefore, the compounded effects of multiple sensory impairments remain less explored.

Notable questions arising from gaps that emerged during this review include the following:

- Does vision screening improve the identification of vision impairments?

- What are the most important visual abilities to screen for?

- What vision screening tools are most reliable, valid, sensitive, specific, and sensible?
- Does remediation of visual impairments triggered by screening improve aspects of functioning (which ones?) and quality of life?

- What should be the targeted outcomes used to evaluate benefit from the remediation of visual impairments?

- How does dementia severity affect each of the above-mentioned questions?

Given the important associations that are emerging between sensory function and cognitive impairment/decline [13], there is now an imperative to apply knowledge concerning vision loss towards the goal of improving vision screening within LTC.

\section{ACKNOWLEDGMENTS}

The database search was conducted by Jessica Babineau, an Information Specialist at the Toronto Rehabilitation Institute-University Health Network. Thanks to M. Kathleen Pichora-Fuller, PhD, Dawn Guthrie, PhD, Kate Dupuis, PhD, CPsych, Jonathan Jarry, MSc, Xochil Argueta-Warden, BScN, RN, and Maxime Grossi, BScN, RN for contributions to this research.

This work was financially supported by the Alzheimer Society Research Programme (ASRP), Alzheimer Society of Canada; grant number RG 1608 and the Walter and Maria Schroeder Institute for Brain Innovation \& Recovery. The sponsors had no role in the conduction of the study or preparation of this manuscript.

Authors' disclosures available online (https:// www.j-alz.com/manuscript-disclosures/18-1129r1).

\section{SUPPLEMENTARY MATERIAL}

The supplementary material is available in the electronic version of this article: http://dx.doi.org/ 10.3233/JAD-181129.

\section{REFERENCES}

[1] Carabellese C, Appollonio I, Rozzini R, Bianchetti A, Frisoni GB, Frattola L, Trabucchi M (1993) Sensory impairment and quality of life in a community elderly population. $J$ Am Geriatr Soc 41, 401-407.

[2] Grue EV, Finne-Soveri H, Stolee P, Poss J, Sörbye LW, Noro A, Hirdes JP, Ranhoff AH (2010) Recent visual decline. A health hazard with consequences for social life: A study of home care clients in 12 countries. Curr Gerontol Geriatr Res 2010, 503817.

[3] Laliberte Rudman D, Gold D, McGrath C, Zuvela B, Spafford MM, Renwick R (2016) "Why would I want to go 
out?": Age-related vision loss and social participation. Can $J$ Aging 35, 465-478.

[4] Lord SR, Dayhew J (2001) Visual risk factors for falls in older people. J Am Geriatr Soc 49, 508-515.

[5] Rovner BW, Ganguli M (1998) Depressive symptoms and disability associated with impaired vision: The MoVIES Project. J Am Geriatr Soc 46, 617-619.

[6] Owsley C1, McGwin G Jr, Scilley K, Meek GC, Seker D, Dyer A (2007) Effect of refractive error correction on healthrelated quality of life and depression in older nursing home residents. Arch Ophthalmol 125, 1471-1477.

[7] Tielsch JM, Javitt JC, Coleman A, Katz J, Sommer A (1995) The prevalence of blindness and visual impairment among nursing home residents in Baltimore. N Engl J Med 332, 1205-1209.

[8] Yamada Y, Vlachova M, Richter T, Finne-Soveri H, Gindin J, van der Roest H, Denkinger MD, Bernabei R, Onder G, Topinkova E (2014) Prevalence and correlates of hearing and visual impairments in European nursing homes: Results from the SHELTER study. J Am Med Dir Assoc 15,738-743.

[9] Chriqui E, Law C, Kergoat MJ, Leclerc BS, Kergoat H (2017) Visual impairment in older institutionalised Canadian seniors with dementia. Ophthalmic Physiol Opt 37, 225-233.

[10] Bowen M, Edgar DF, Hancock B, Haque S, Shah R, Buchanan S, Iliffe S, Maskell S, Pickett J, Taylor JP, O-Leary N (2016) The prevalence of visual impairment in people with dementia (the PrOVIDe study): A cross-sectional study of people aged 60-89 years with dementia and qualitative exploration of individual, career and professional perspectives. NIHR Journals Library, Southampton, UK.

[11] Guthrie DM, Davidson JGS, Williams N, Campos J, Hunter K, Mick P, Orange JB, Pichora-Fuller MK, Phillips NA, Savundranayagam MY, Wittich W (2018) Combined impairments in vision, hearing and cognition are associated with greater levels of functional and communication difficulties than cognitive impairment alone: Analysis of interRAI data for home care and long-term care recipients in Ontario. PLoS One, 13, e0192971.

[12] Whitson HE, Cousins SW, Burchett BM, Hybels CF, Pieper CF, Cohen HJ (2007) The combined effect of visual impairment and cognitive impairment on disability in older people. $J$ Am Geriatr Soc 55, 855-891.

[13] Albers MW, Gilmore GC, Kaye J, Murphy C, Wingfield A, Bennett DA, Boxer AL, Buchman AS, Cruickshanks KJ, Devanand DP, Duffy CJ, Gall CM, Gates GA, Cranholm AC, Hensch T, Holtzer R, Hyman BT, Lin FR, McKee AC, Morris JC, Petersen RC, Silbert LC, Struble RG, Trojanowski JQ, Verghese J, Wilson DA, Xu S, Zhang LI (2015) At the interface of sensory and motor dysfunctions and Alzheimer's disease. Alzheimers Dement 11, 70-98.

[14] Chen SP, Bhattacharya J, Pershing S (2017) Association of vision loss with cognition in older adults. JAMA Ophthalmol 135, 963-970.

[15] Cronin-Golomb A, Corkin S, Growdon JH (1995) Visual dysfunction predicts cognitive deficits in Alzheimer's disease. Optom Vis Sci 72, 168-176.

[16] Frost S, Martins RN, Kanagasingam Y (2010) Ocular biomarkers for early detection of Alzheimer's disease. $J$ Alzheimers Dis 22, 1-16.

[17] Humes LE, Busey TA, Craig J, Kewley-Port D (2013) Are age-related changes in cognitive function driven by age-related changes in sensory processing? Atten Percept Psychophys 75, 508-524.
[18] Jackson GR, Owsley C (2003) Visual dysfunction, neurodegenerative diseases, and aging. Neurol Clin 21, 709-728.

[19] Jefferis JM, Mosimann UP, Clarke MP (2011) Cataract and cognitive impairment: A review of the literature. $\mathrm{Br} \mathrm{J} \mathrm{Oph-}$ thalmol 95, 17-23.

[20] Salthouse TA, Hancock HE, Meinz EJ, Hambrick DZ (1996) Interrelations of age, visual acuity, and cognitive functioning. J Gerontol B Psychol Sci Soc Sci 51, 317-330.

[21] Valenti DA (2010) Alzheimer's disease: Visual system review. Optometry 81, 12-21.

[22] Horowitz A (1997) The relationship between vision impairment and the assessment of disruptive behaviors among nursing home residents. Gerontologist 37, 620-628.

[23] Dickinson C, Rabbitt P (1991) Simulated visual impairment: Effects on text comprehension and reading speed. Clinic Vis Sci 6, 301-308.

[24] Armstrong R, Kergoat H (2015) Oculo-visual changes and clinical considerations affecting older patients with dementia. Ophthalmic Physiol Opt 35, 352-376.

[25] Canevelli M, Valletta M, Trebbastoni A, Sarli G, D’Antonio F, Tariciotti L, de Lena C, Bruno G (2016) Sundowning in dementia: Clinical relevance, pathophysiological determinants, and therapeutic approaches. Front Med 3, 73.

[26] Coleman AL, Yu F, Keeler E, Mangione CM (2006) Treatment of uncorrected refractive error improves visionspecific quality of life. J Am Geriatr Soc 54, 883-890.

[27] Jefferis JM, Clarke MP, Taylor JP (2015) Effect of cataract surgery on cognition, mood, and visual hallucinations in older adults. J Cataract Refract Surg 41, 1241-1247.

[28] Owsley C, McGwin Jr G, Scilley K, Meek GC, Seker D, Dyer A (2007) Impact of cataract surgery on health-related quality of life in nursing home residents. Br J Ophthalmol 91, 1359-1363.

[29] McGilton KS, Höbler F, Campos J, Dupuis K, Labreche T, Guthrie DM, Jarry J, Singh G, Wittich W (2016) Hearing and vision screening tools for long-term care residents with dementia: Protocol for a scoping review. BMJ Open 6 , $\mathrm{e} 011945$

[30] Höbler F, Argueta-Warden X, Rodríguez-Monforte M, Escrig-Pinol A, Wittich W, McGilton KS (2018) Exploring the sensory screening experiences of nurses working in long-term care homes with residents who have dementia: A qualitative study. BMC Geriatr 18, 235.

[31] Wittich W, Höbler F, Jarry J, McGilton KS (2018) Recommendations for successful sensory screening in older adults with dementia in long-term care: A qualitative environmental review. BMJ Open 8, e019451.

[32] Feder K, Michaud D, Ramage-Morin P, McNamee J, Beauregard Y (2015) Prevalence of hearing loss among Canadians aged 20 to 79: Audiometric results from the 2012/2013 Canadian Health Measures Survey. Health Rep 26, 18-25.

[33] Lin FR, Metter EJ, O'Brien RJ, Resnick SM, Zonderman AB, Ferrucci L (2011) Hearing loss and incident dementia. Arch Neurol 68, 214-220.

[34] Lin FR (2011) Hearing loss and cognition among older adults in the United States. J Gerontol A Biol Sci Med Sci 66, 1131-1136.

[35] Lin FR, Yaffe K, Xia J, Xue QL, Harris TB, PurchaseHelzner E, Satterfield S, Ayonayon HN, Ferrucci L, Simonsick EM, Health ABC Study Group (2013) Hearing loss and cognitive decline in older adults. JAMA Intern Med 173, 293-299.

[36] Kergoat H, Boisjoly H, Freeman EE, Monette J, Roy S, Kergoat MJ (2014) The perceived needs and availability of 
eye care services for older adults in Quebec long-term care facilities. Can Geriatr J 17, 108-113.

[37] Kergoat H, Law C, Chriqui E, Leclerc BS, Kergoat MJ (2017) Tool for screening visual acuity in older individuals with dementia. Am J Alzheimers Dis Other Demen 32, 96-100.

[38] Whiteside MM, Wallhagen MI, Pettengill E (2006) Sensory impairment in older adults: Part 2: Vision loss. Am J Nurs 106, 52-61.

[39] Phillips CD, Chu CW, Morris JN, Hawes C (1993) Effects of cognitive impairment on the reliability of geriatric assessments in nursing homes. J Am Geriatr Soc 41, 136-142.

[40] Arksey H, O'Malley L (2005) Scoping studies: Towards a methodological framework. Int J Soc Res Methodol 8, 19-32.

[41] Feinstein AR (1987) The theory and evaluation of sensibility. In Clinimetrics, Feinstein AR, ed. Murray Printing Company, Westford, MA, pp. 141-166.

[42] Thibault L, Kergoat H (2016) Eye care services for older institutionalised individuals affected by cognitive and visual deficits: A systematic review. Ophthalmic Physiol Opt 36, 566-583.

[43] Jee J, Wang JJ, Rose KA, Lindley R, Landau P, Mitchell P (2005) Vision and hearing impairment in aged care clients. Ophthalmic Epidemiol 12, 199-205.

[44] Caban AJ, Lee DJ, Gomez-Marin O, Lam BL, Zheng DD (2005) Prevalence of concurrent hearing and visual impairment in US adults: The national health interview survey, 1997-2002. Am J Public Health 95, 1940-1942.

[45] Roets-Merken L, Zuidema S, Vernooij-Dassen M, Dees M, Hermsen P, Kempen G, Graff M (2017) Problems identified by dual sensory impaired older adults in long-term care when using a self-management program: A qualitative study. PLoS One 12, e0173601.

[46] Yamada Y, Denkinger MD, Onder G, Henrard JC, van der Roest HG, Finne-Soveri H, Richter T, Vlachova M, Bernabei R, Topinkova E (2016) Dual sensory impairment and cognitive decline: The results from the shelter study. J Gerontol A Biol Sci Med Sci 71, 1-7.

[47] Yamada Y, Denkinger MD, Onder G, Finne-Soveri H, van der Roest H, Vlachova M, Richter T, Gindin J, Bernabei R, Topinkova E (2015) Impact of dual sensory impairment on onset of behavioral symptoms in European nursing homes: Results from the Services and Health for Elderly in LongTerm Care study. J Am Med Dir Assoc 16, 329-333.

[48] Ferris 3rd FL, Kassoff A, Bresnick GH, Bailey I (1982) New visual acuity charts for clinical research. Am J Ophthalmol 94, 91-96.

[49] Snellen H (1862) Optotypi ad visum determinandum. van de Weijer PM, ed. Utrecht.

[50] Landolt E (1891) Nouvelles recherches sur la physiologie des mouvements des yeux. Arch d'ophthalmologie 11, 385395.

[51] Ishihara S (1917) Tests for color-blindness. Am J Ophthalmol 1, 457.

[52] Fletcher R (1980) The City University Colour Vision Test, Keeler, England.

[53] Mangione CM, Lee PP, Pitts J, Gutierrez P, Berry S, Hays RD (1998) Psychometric properties of the National Eye Institute Visual Function Questionnaire (NEI-VFQ), NEI-VFQ Field Test Investigators. Arch Ophthalmol 116, 1496-150. 